\title{
The Critical Conditions for Thermal Explosion in a System Heated at a Constant Rate.
}

\author{
D. Sánchez-Rodriguez, J. Farjas*, P. Roura. \\ University of Girona, Campus Montilivi, Edif. PII, E17071 Girona, Catalonia, Spain
}

\begin{abstract}
We have analyzed the condition needed for thermal explosion to occur in a solid sample when the temperature of the vessel walls is raised at a constant rate. We have developed a dimensionless model that allows its direct comparison with an isoperibolic system (constant vessel wall temperature). We have obtained an analytical expression for the critical condition as a function of the system parameters. Our solution takes into account reactant consumption and covers different geometries: thin film, finite and infinite cylinder. The critical condition has been validated with numerical simulations and experiments. We show that, compared to the isoperibolic system, thermal explosion is a little bit more difficult to achieve under constant heating conditions. Besides, we show that thermal explosion on submicrometric films is nearly impossible.
\end{abstract}

Keywords: Thermal explosion, thermal runaway, volume combustion synthesis, ignition condition, thin-film combustion synthesis, gasless reactions.

*Corresponding author: jordi.farjas@udg.edu, Tel (34)972418490, Fax (34) 972418098 University of Girona, Campus Montilivi, Edif. PII, E17071 Girona, Catalonia, Spain 


\section{Nomenclature and Units}

$A \quad$ Pre-exponential constant, $\mathrm{s}^{-1}$

$a \quad$ Thermal diffusivity, $\mathrm{m}^{2} / \mathrm{s}$

$b \quad$ Heating rate, Eq. (3), K/s

$c \quad$ Specific heat capacity, $\mathrm{J} /(\mathrm{kg} \cdot \mathrm{K})$

C Geometrical factor of the critical condition, Eq. (15).

$d \quad$ characteristic linear dimension of Frank-Kamenetskii parameter, Eq. (13), m

$d_{i} \quad$ Width of the reaction zone, Eq. (9), m

$E_{A} \quad$ Activation energy, Eq. (4), J/mol

$H \quad$ Sample height or film thickness

$m \quad$ Sample mass, $\mathrm{kg}$

$N \quad$ coefficient related to the geometry of the diffusion equation, Eq. (10).

$q \quad$ Specific heat of reaction (positive for exothermic reactions), $\mathrm{J} / \mathrm{kg}$

$R_{G} \quad$ Universal gas constant, $\mathrm{J} /(\mathrm{K} \cdot \mathrm{mol})$

$R \quad$ Inner radius of a cylindrical or spherical reaction vessel

$r \quad$ Radial space coordinate, $\mathrm{m}$

$t \quad$ Time, $\mathrm{s}$

$t_{R} \quad$ Reaction time scale, Eq. (5), $\mathrm{s}$

$t_{i} \quad$ Time scale of the adiabatic induction period, Eq. (8), $\mathrm{s}$

$T \quad$ Temperature, $\mathrm{K}$

$T_{f} \quad$ Temperature of the vessel containing the system, $\mathrm{K}$

$T_{\text {Kis }} \quad$ Maximum rate transformation temperature, Eq. (6), $\mathrm{K}$

$V \quad$ System's volume, $\mathrm{m}^{3}$

$z \quad$ Vertical space coordinate, $\mathrm{m}$

$\lambda \quad$ Thermal conductivity, $\mathrm{W} /(\mathrm{m} \cdot \mathrm{K})$

$\rho \quad$ Density, $\mathrm{kg} / \mathrm{m}^{3}$

\section{Dimensionless parameters}

$\alpha \quad$ Degree of transformation or conversion fraction

$\bar{\alpha} \quad$ Volume averaged degree of transformation, Eq. (14).

$\varepsilon \quad$ Arrhenius parameter, Eq. (13).

$\delta \quad$ Frank-Kamenetskii parameter, Eq. (13).

$\xi \quad$ Space coordinate, Eq. (7). 
$\theta \quad$ Temperature, Eq. (7).

$\theta_{T} \quad$ Todes parameter, Eq. (13).

$\tau \quad$ Time, Eq. (7).

\section{Subscripts and superscripts}

cr critical, parameter value at the combustion threshold

in initial

fin final

(1D) $1 \mathrm{D}$ model

(2D) 2D model

TF 1D vessel geometry in the limit case $R>>H$ (thin film or semi-infinite slab)

Cyl 1D vessel geometry in the limit case $H \gg>R$ (infinite cylinder)

Sph Spherical vessel 


\section{Introduction}

Knowledge of the critical conditions for the occurrence of thermal explosion (sample mass, container geometry, activation energy, thermal conductivity, temperature) allows to determine: a) the chemical risk associated with the storage and transportation of hazardous materials or in running chemical reactors [1-6], b) the conditions for pyrotechnic reactions to occur [7-9], c) munitions cookoff temperatures [10] and d) in general the ignition condition in chemical engineering processes [11,12].

Furthermore, the so-called "combustion synthesis" takes advantage of local heating related to the heat evolved during the chemical reaction to obtain materials at low processing temperature $[13,14]$. For instance, synthesis of functional metal oxide thin-films via combustion synthesis has attracted great attention because it would allow the use of low-temperature substrates and it would be a promising route towards the development of large-area and low-cost printed electronics [15]. Unfortunately, under isothermal conditions or during slow heating ramps, fast heat dissipation to the substrate hinders thermal explosion by avoiding the local overheating needed for a thermal runaway to occur [16-22]. Several authors have suggested that, during rapid heating ramps, combustion may be achieved in thin films $[14,16]$. So, the conditions leading to thermal runaway during heating at a constant rate is of interest for many applications.

In [23-28] thermal explosion under dynamic conditions is analysed numerically and theoretically. The parameter range explored numerically is quite limited. Besides, to reduce the original system to an ordinary differential equation (ODE) system, analytical approaches neglect the temperature and transformation degree distribution. As we will see, this approximation is too crude to provide an accurate description of the critical condition. As Boddington et al. [29] noted for the constant heating case: "The development of a realistic model for the ignition experiment is a formidable problem."

The aim of the present work is to provide a thermal runaway condition for a system that is heated uniformly at a constant rate. To be more precise, the reactant is placed in a vessel whose walls are kept at a temperature, $T_{f}$, that is raised at a constant rate. We consider a homogeneous solid sample where heat dissipation occurs mainly through heat diffusion towards the vessel walls. We will show that this model correctly describes the observed behavior of one metalorganic precursor. We will use a new dimensionless system of equations that is equivalent to that introduced by FrankKamenetskii for an isoperibolic system (a system where $T_{f}$ remains constant with time) 
[30,31]. Thus, our approach allows to determine whether thermal runaway during constant heating is easier or not than during an isothermal temperature program. We will obtain a combustion condition that covers different geometries and a wide parameter range that accounts for most practical cases. Finally, we will analyze the possibility of combustion in thin films.

\section{The model}

The model is based on the classical theory for ignition and front propagation in solid samples $[11,21,30,32-35]$. The heat balance is the result of two opposite effects: heat generation by chemical reaction and heat removal through thermal conduction. We neglect the effect of reactive gas depletion or the evolution of the system parameters during the reaction. These are reasonable approximations to predict the development of a thermal runaway but are not sufficient to accurately describe the whole reaction course. We also assume a homogeneous medium. The last assumption is also valid for heterogeneous systems provided that the time of heat exchange is much shorter than that of the chemical reaction [36]. For a cylindrical vessel, the evolution of the temperature is described by a two dimensional (2D) partial differential equation (PDE):

$$
\rho c \frac{\partial T}{\partial t}=\lambda\left[\frac{1}{r} \frac{\partial}{\partial r}\left(r \frac{\partial T}{\partial r}\right)+\frac{\partial^{2} T}{\partial z^{2}}\right]+\rho q \frac{\partial \alpha}{\partial t},
$$

where $\rho$ is density, $c$ is heat capacity, $q$ is the reaction specific heat and $\lambda$ is thermal conductivity; $T$ is temperature, $t$ is time, $z$ and $r$ are the axial and radial coordinates, respectively (see Fig. 1), and $\alpha(r, z, t)$ is the transformation degree ( $\alpha=0$ untransformed, $\alpha=1$ totally transformed).

We assume that the initial sample temperature, $T_{i n}$, is uniform and low enough to ensure that the degree of transformation is zero throughout the sample. The boundary conditions are:

$$
\left.\frac{\partial T}{\partial r}\right|_{r=0}=0, \quad T(R, z)=T_{f}, \quad T(r, 0)=T_{f},\left.\quad \frac{\partial T}{\partial z}\right|_{z=H}=0,
$$

where $H$ is the sample height, $R$ is the vessel's inner radius and $T_{f}$ is the vessel temperature, that is raised at a constant rate $b$ :

$$
T_{f}=T_{i n}+b t
$$


The last boundary condition states that the heat flux is null at the top of the sample. Actually, if the vessel temperature is below $600^{\circ} \mathrm{C}$, the heat lost by radiation is negligible when compared to the heat dissipated by conduction through the crucible walls. As for the heat evolved from the released gases, it is nearly compensated by the loss of heat capacity; so, it has a negligible effect on the heat balance [37]. Finally, due to symmetry, this boundary condition also describes a closed vessel full of reactant provided that $H$ is the vessel half height.

Concerning the reaction, we assume first-order reaction kinetics to account for the reactant consumption and an Arrhenian temperature dependence [38,39]:

$$
\frac{\partial \alpha}{\partial t}=A e^{-E_{A} / R_{G} T}(1-\alpha),
$$

where $A$ and $E_{A}$ are the pre-exponential constant and the activation energy of the reaction rate constant, respectively, and $R_{G}$ is the universal gas constant.

\subsection{Dimensionless system}

The analysis of the system becomes much simpler by introducing suitable dimensionless parameters. Previous analytical studies have based their dimensionless model on the critical temperature of the isoperibolic system (static conditions) [24-27]. To derive a model that is formally similar to the isoperibolic system [21], we have looked for a different normalization.

The only difference with respect to the isoperibolic system is the second and third boundary equations: in the isoperibolic system the vessel temperature remains constant while in the present case it increases steadily at rate $b$. If overheating due to the reaction is negligible, the reaction time, $t_{R}$, is determined by the vessel temperature; it is inversely proportional to the rate constant. Under constant heating conditions, it has been shown that the reaction time has the same functional dependence but on $T_{K i s}$ [40,41],

$$
t_{R}=\left(A e^{-E_{A} / R_{G} T_{\text {Kis }}}\right)^{-1},
$$

where $T_{K i s}$ is the temperature at which the reaction rate is at its maximum, and is given by Kissinger's equation $[42,43]$ :

$$
\frac{E_{A}}{R_{G} T_{K i s}^{2}}=\frac{A}{b} e^{-E_{A} / R_{G} T_{K i s}} .
$$


Since the role played by the Kissinger temperature, $T_{K i s}$, for constant heating rate is similar to the constant vessel temperature for the isoperibolic system, it seems natural to use the same dimensionless temperature, $\theta$, time, $\tau$, and space coordinate, $\xi$, already defined for the isoperibolic system [30,31] but replacing $T_{f}$ by $T_{K i s}$. For the particular 1D case of a thin film we thus define:

$$
\theta \equiv \frac{E_{A}}{R_{G} T_{K i s}^{2}}\left(T-T_{K i s}\right), \tau \equiv \frac{t}{t_{i}} \text { and } \xi \equiv \frac{z}{d_{i}},
$$

where, $t_{i}$

$$
t_{i} \equiv\left(\frac{q}{c} \frac{E_{A}}{R_{G} T_{K i s}^{2}} A e^{-\frac{E_{A}}{R_{G} T_{K i s}}}\right)^{-1}=\left(\frac{q}{c} \frac{b E_{A}^{2}}{R_{G}^{2} T_{K i s}^{4}}\right)^{-1}
$$

and $d_{i}$

$$
d_{i} \equiv \sqrt{\frac{\lambda}{\rho c} t_{i}}
$$

The length and time scales $d_{i}$ and $t_{i}$ have a precise meaning for the isoperibolic system: $d_{i}$ is the width of the zone were the reaction rate is significant [30,31] and $t_{i}$ is the time elapsed before the thermal runaway [44]. With the above dimensionless variables, Eqs.(1) and (4) become for a thin film:

$$
\begin{aligned}
& \frac{\partial \theta}{\partial \tau}=\left(\frac{\partial^{2} \theta}{\partial \xi^{2}}+\frac{N}{\xi} \frac{\partial \theta}{\partial \xi}\right)+\theta_{T} \frac{\partial \alpha}{\partial \tau} \\
& \frac{\partial \alpha}{\partial \tau}=\frac{1}{\theta_{T}} e^{\frac{\theta}{1+\varepsilon \theta}}(1-\alpha)
\end{aligned},
$$

where $N=0$ with the boundary conditions:

$$
\left.\frac{\partial \theta}{\partial \xi}\right|_{\xi=0}=0 \quad \text { and } \quad \theta(\sqrt{\delta})=\frac{\tau}{\theta_{T}}
$$

The initial conditions are,

$$
\theta_{i n}=-\frac{1}{\varepsilon} \text { and } \tau_{\text {in }}=-\frac{\theta_{T}}{\varepsilon},
$$

where we have set $t=0$ when $T_{K i s}$ is reached. We have taken $T_{i n}=0 \mathrm{~K}$ so that the condition that initially the transformation rate is null is fulfilled.

Eqs. (10)-(13) reveal that the 1D system is fully described by three dimensionless parameters:

$$
\varepsilon \equiv \frac{R_{G} T_{K i s}}{E_{A}}, \theta_{T} \equiv \frac{E_{A}}{R_{G} T_{K i s}^{2}} \frac{q}{c}, \delta \equiv \rho \frac{q}{\lambda} \frac{E_{A}}{R_{G} T_{K i s}^{2}} d^{2} A e^{-\frac{E_{A}}{R_{G} T_{K i s}}}=\left(\frac{d}{d_{i}}\right)^{2}
$$


where $d$ for a thin film is the height $(d=H)$. We will call these parameters the Ahrrenius $(\varepsilon)$, Todes $\left(\theta_{T}\right)$ and Frank-Kamenetskii $(\delta)$ parameters to keep the same nomenclature than for the isoperibolic system.

Generalization of the above equations is straightforward for other 1D geometries: for a sphere $(N=2$ and $d=R)$ and for an infinite cylinder $(N=1, d=R)$. The derivation of the 2D system is straightforward from Eqs. (1)-(4) and (7) but, for the sake of simplicity, in the analysis of the critical condition we have preferred to write down only the 1D model. Nevertheless, the 2D simulations presented below for a cylinder or radius $R$ and height $H$ have been obtained from the numerical solution of the dimensionless model. In this case: $\frac{1}{d}=\frac{1}{H}+\frac{1}{R}[21]$.

\section{Experimental and numerical results}

To assess the ability of the numerical model to describe real situations, we have analyzed numerically and experimentally the decomposition of barium trifluoroacetate, $\mathrm{Ba}\left(\mathrm{CF}_{3} \mathrm{COO}\right)_{2}\left[\mathrm{Ba}(\mathrm{TFA})_{2}\right]$. Preparation details are given in ref. [45]. The evolution of the reaction with the temperature is monitored by thermogravimetry (TG). TG measures the evolution of the mass of a sample when it is submitted to a controlled temperature program. The conversion fraction at any time can be easily calculated as [46]:

$$
\bar{\alpha}(t) \equiv \frac{1}{V} \int_{V} \alpha d V \approx \frac{m_{i n}-m_{t}}{m_{i n}-m_{f i n}} .
$$

where $V$ is the system's volume, $m_{i n}$ is the initial sample mass, $m_{t}$ is the mass at time $t$ and $m_{\text {fin }}$ is the final mass.

TG analysis was performed with a Mettler Toledo model TGA851eLF thermobalance. Samples were placed inside uncovered alumina crucibles. The internal and external radii of the crucible were 2.4 and $3 \mathrm{~mm}$, respectively. Measurements were corrected with a blank experiment carried out under identical conditions. Inside the furnace a gas flow rate of $50 \mathrm{~mL} / \mathrm{min}$ was controlled by mass flow meters. High purity synthetic air was used. Water-saturated air was obtained by bubbling the carrier gas in water at standard temperature and pressure $\left(25^{\circ} \mathrm{C}, 1 \mathrm{~atm}\right)$. Thermal analysis experiments were performed at $20 \mathrm{~K} / \mathrm{min}$. The parameters used to simulate the thermal decomposition $\mathrm{Ba}(\mathrm{TFA})_{2}$ are summarized in Table 1. All have been measured and the details are given in ref. [47]. 
We can see in Fig. 2 an abrupt step in the evolution of $\bar{\alpha}$ for $m_{\text {in }}=16 \mathrm{mg}$ that reveals the occurrence of a thermal runaway [17,22,47,29,48]. Movie 1 shows the evolution of $\alpha$. Vertical and horizontal axes correspond to the $z$ and $r$ coordinates, respectively (see Fig. 1); so, the top left corner corresponds to the top center point in the sample. Given that, when a thermal runaway occurs, the decomposition rate increases enormously, frames are taken at constant degree of transformation intervals; otherwise, decomposition would be like a flash in the movie. From Movie 1, we can realize that a front is initiated at the top center. The reason is that the vessel walls are a thermal sink that allows rapid heat dissipation thus preventing local overheating and, consequently, the development of a thermal runaway there. A thermal runaway occurs when the sample size is greater than a critical value. Consequently, for sample sizes near the critical value, the thermal runaway is always initiated at the farthest location from the vessel walls, i.e., the top center of the sample, and propagates towards the vessel walls. For large samples, the thermal runaway is initiated next to the vessel walls at a distance of the order of $d_{i}$ and propagates towards the center of the sample. This behavior is illustrated in Movie 2. Therefore, for large chemical reactors, the front begins very close to the reactor walls and propagates from the reactor walls to the center of the sample. For intermediate sizes (see Movie 3) two fronts propagate in opposite directions. This displacement of the ignition point from the center of the sample to the walls as the width of the sample increases was also reported by Merzhanov et al. [31] in a isoperibolic system.

Since $\mathrm{Ba}(\mathrm{TFA})_{2}$ decomposition is governed by the superposition of two different mechanisms [45], the assumption that the reaction is ruled by a first order reaction is quite inaccurate. Thus, we would expect our numerical model to provide a rather poor prediction of the time evolution of $\bar{\alpha}$. In Fig. 2 we have plotted simulated and experimental $\mathrm{Ba}(\mathrm{TFA})_{2}$ transformation curves when the sample is heated at $20 \mathrm{~K} / \mathrm{min}$ for different sample masses. Although our model fails to predict $\bar{\alpha}$ accurately, it does provide a rather good description of the first stages of the reaction, which is sufficient for our purpose of establishing the required conditions for a thermal runaway to develop. In the following, we will predict the critical condition for a thermal runaway to occur.

\section{Critical condition.}


As already stated in Section 2, the dimensionless set of equations describing the evolution of a 1D system, Eqs. (10)-(12), depends only on three parameters; namely $\varepsilon$, $\theta_{T}$ and $\delta$, Eq. (13). They collect all the physical parameters concerning the chemical reaction, heat transport, size (through $d$ ) and heating rate (implicitly through $T_{\text {Kis }}$ ). Depending on the particular $\varepsilon, \theta_{T}$ and $\delta$ values, the system will undergo thermal explosion or not. Our aim is to find the boundary of the $\varepsilon, \theta_{T}$ and $\delta$ region where combustion occurs. This boundary is described by a so called "critical condition" relating these three parameters. In the limit of very exothermic transformations and high activation energies $\left(\theta_{T} \rightarrow \infty\right.$ and $\varepsilon \rightarrow 0$ ), the critical condition states that $\delta_{c r}(\delta$ in the boundary) equals a constant value that depends on geometry.

Two general analytical approaches are used to simplify the system. In the quasistationary approach, the subcritical regime is related to the existence of a quasistationary solution where the temperature evolution closely follows the heating rate. The quasi-stationary solution allows deriving the spatial dependence of $\alpha$ and $T$ in the subcritical regime. Conversely, in the non-stationary approach, the PDE is reduced to an ODE system. The resulting ODE system allows calculating the time evolution of the averaged $\alpha$ and $T$. Both approaches have been applied to the isoperibolic case in our former paper [21] and are applied here to the constant heating case. The technical developments are detailed in Appendices A and B.

\subsection{Exact solution}

We have numerically integrated the exact model, Eqs. (10)-(12), for the particular case of a thin film. We have calculated the evolution of the heat released by the reaction and the heat dissipated by thermal conduction towards the substrate. Their ratio is a good criterion to determine whether thermal runaway develops or not [21]. We have used this criterion because it can be applied to both the exact model and the non-stationary approach. In particular, for a given set of parameters $\theta_{T}$ and $\varepsilon$, we look for the value of $\delta$ at the threshold of thermal runaway. The result is shown in Fig. 3 where the value of $\delta_{c r}$ is plotted as a function of $\theta_{T}$ for several values of $\varepsilon$.

We should emphasize that the range of parameters in Fig. $3\left(10<\theta_{T}<10^{4}, 0<\varepsilon\right.$ $<0.1$ ) covers most practical situations. Most bond energies are between 50 and 500 
$\mathrm{kJ} / \mathrm{mol}$ (bond energies bellow $50 \mathrm{~kJ} / \mathrm{mol}$ are characteristic of weak interactions such as Van der Waals bonding). Assuming $T_{K i s}=600 \mathrm{~K}$, a variation of $E_{\mathrm{A}}$ between 50 and 500 $\mathrm{kJ} / \mathrm{mol}$ results in a variation of $\varepsilon$ between 0.01 and 0.1 . As for $\theta_{T}$ the quotient $q / c$ corresponds to the adiabatic temperature rise. The adiabatic temperature rise is typically around $1000 \mathrm{~K}[13,49]$. However, in the case of metalorganic precursors, combustion has been observed in powders for $q / c$ values of few hundred Kelvins [17,45,50,51]. If we assume that the adiabatic temperature rise is at least of the same magnitude than $T_{K i s}$ we obtain a lower bound for $\theta_{T}$ of 10 (for a maximum value of $\varepsilon=0.1$ ).

\subsection{Non-stationary approach}

The base of the existing analytical solutions is the set of ODE obtained after neglecting the temperature distribution in the system's volume $[23,24,26]$. Using this approach, in Appendix B we derive the ODE system,

$$
\begin{aligned}
& \frac{d \theta}{d \tau}=-\frac{e C}{\delta}\left(\theta-\frac{\tau}{\theta_{T}}\right)+\theta_{T} \frac{d \alpha}{d \tau} \\
& \frac{d \alpha}{d \tau}=\frac{1}{\theta_{T}} e^{\frac{\theta}{1+\varepsilon \theta}}(1-\alpha)
\end{aligned}
$$

where $C$ is a constant that depends on the system's geometry: $C_{S p h e}^{(1 D)}=3.32$ for a spherical vessel [52], $C_{C y l}^{(1 D)}=2.0$ for an infinite cylinder [30,53,54], $C_{T F}^{(1 D)}=0.878$ for a thin film $[30,53,54]$. The initial conditions are those of Eq. (12).

In Fig. 3 we compare the critical value of Frank-Kamenetskii parameter, $\delta_{\text {cr }}$, obtained from the numerical integration of the exact model, Eqs. (10)-(12), with the value obtained from the ODE system, Eq. (15). From Fig. 3 it is apparent that the ODE system correctly describes the functional dependence of $\delta_{\mathrm{cr}}$ on $\theta_{T}$ and $\varepsilon$ but, in contrast with the isoperibolic case [21], it fails to deliver an accurate prediction of the $\delta_{c r}$ value. This is because, in the dynamic case, neglecting the temperature and degree transformation distribution is an approximation to rough to obtain an accurate prediction of the combustion threshold. Since the quasi-stationary model (Appendix A) takes into account the temperature distribution, we derive the critical condition combining analytical results of the stationary model with numerical results.

\subsection{The critical condition}


To simplify the fitting procedure, we take advantage of the results delivered by the quasi-stationary approach (See Appendix A) and the fact that, for any value of $\varepsilon, \delta_{c r}$ tends to a constant value in the limit $\theta_{T} \rightarrow \infty$. These asymptotic values have been fitted to $\delta=4.392 /(1+2 \varepsilon)$, see inset of Fig. 3. The proportionality constant is the value of $\delta$ deduced for $\varepsilon=0$ in Appendix A:

$$
\delta_{c r}=5 C_{T F}=4.392 \quad\left(\varepsilon=0, \theta_{T} \rightarrow \infty\right) .
$$

Eq. (16) and the critical condition for an isoperibolic system [21,30] are nearly identical, the main difference is a factor 5 that is related to reactant consumption prior to thermal runaway. At the threshold, thermal runaway develops when the transformation rate at the top center of the sample reaches its maximum value, and this occurs for the Kissinger temperature. Due to the thermal gradient between the center and the vessel walls, the thermal runaway is achieved for a furnace temperature below the Kissinger one (see Appendix A):

$$
T_{f, c r}=T_{K i s}(1-\varepsilon) .
$$

Since in most real system $\varepsilon<<1$, the furnace critical temperature approaches the Kissinger one. Besides, the initial reactant consumption makes thermal explosion a little bit more difficult to achieve under constant heating conditions for a given temperature ( $T_{f}$ for the static case or $T_{\text {Kis }}$ for the dynamic case).

The shape of the $\delta\left(\theta_{T}\right)$ curves can be fitted to the functional dependence $\delta_{c r} \propto\left(1-f(\varepsilon) / \theta_{T}\right)^{-1}$ where $f(\varepsilon)$ has been expanded up to $\varepsilon^{2}$ term. The best fit to $\delta_{c r}$ for a thin film has been obtained with $f(\varepsilon)=2\left(2+\varepsilon+30 \varepsilon^{2}\right)$, see Fig. 4.a. Finally, the particular critical condition for the thin film can be generalized to the other geometries by replacing $C_{T F}$ by the corresponding $C$ :

$$
\left.\delta(1+2 \varepsilon)\left\{1-\left(2+\varepsilon+30 \varepsilon^{2}\right) \frac{2}{\theta_{T}}\right\}\right|_{c r}=5 C .
$$

This generalization is reasonable because it has been shown, from the non-stationary analysis of an isoperibolic system, that the contribution of geometry can be separated from the rest of parameters [21]. In the present case, the non-stationary analysis agrees approximately with the exact dependence of $\delta_{c r}$ on $\theta_{T}$ and $\varepsilon$; so, we consider that geometry can also be separated. 
To check the accuracy of the critical condition, the prediction of $\delta_{c r}$ delivered by Eq. (18) has been compared to the exact value obtained by numerical integration of the PDE system for two 1D geometries: thin film, Fig. 4a, and infinite cylinder, Fig. 4b. In all these cases, the prediction (dashed lines) departs less than $2 \%$ from the exact value.

For the isoperibolic system, the critical condition is also valid for the $2 \mathrm{D}$ geometry of a finite cylinder [21]. In that case, $C$ must be simply replaced by:

$$
C_{c y l}^{2 D}=\frac{0.878 R^{2}+2 H^{2}}{(H+R)^{2}} .
$$

To test that this assumption works under constant heating conditions, the exact 2D model for finite cylinder, Eqs. (1)-(4), has been numerically solved and the left-hand side of Eq. (18) has been calculated for the $\varepsilon, \theta_{T}$ and $\delta$ values at the combustion boundary. The results are plotted as symbols in Fig. 5 and compared with $C_{C y l}^{(2 D)}$ of Eq. (19). The agreement is excellent.

\subsection{Critical mass for a finite size cylindrical vessel.}

Combining Eqs. (18), (19) and the definition of $\delta$, Eq. (13), one can determine the sample critical mass:

$$
m_{c r}=5 \pi \rho a \frac{t_{R}}{\theta_{T}} \frac{0.878 R^{2}+2 H^{2}}{H(1+2 \varepsilon)\left\{1-\left(2+\varepsilon+30 \varepsilon^{2}\right) \frac{2}{\theta_{T}}\right\}}
$$

where $a$ is thermal diffusivity ( $a \equiv \lambda / \rho c$ ) and $t_{R}$ is the reaction time, Eq. (5). Eq. (20) has been obtained assuming that the vessel containing the sample is not covered by a lid. The case of a sample that fills a closed vessel is also described by Eq. (20) but with $H$ being half the sample height and multiplying the right hand side of Eq. (20) by a factor of 2 .

The predicted critical sample mass for $\mathrm{Ba}(\mathrm{TFA})_{2}$ can be calculated as follows. Eq. (20) can be solved for $H$ if we substitute $m_{c r}$ by:

$$
m=\rho \pi R^{2} H
$$

and calculate the physical parameters from those of table $1, R=2.4 \mathrm{~mm}$ and $b=20$ $\mathrm{K} / \mathrm{min}$. The obtained critical height $(0.501 \mathrm{~mm})$ corresponds to a critical mass of 13.3 mg. In Fig. 3, the experimental curve for $m_{\text {in }}=16 \mathrm{mg}$ exhibits the abrupt mass loss step characteristic of a thermal runaway while for $m_{\text {in }}=11 \mathrm{mg}$ this is not observed. A similar 
prediction is obtained by the exact numerical integration of the PDE system: the transition from a smooth to a sharp evolution takes place between 13 and $16 \mathrm{mg}$. So, we have achieved nice agreement between experiments, numerical simulations and the critical condition, Eq. (20).

\section{Combustion in thin films}

Despite the claim that metal organic precursor films can be transformed into oxide films at low processing temperatures thanks to thermal explosion [15], we have been unable to reproduce these results so far [17]. In fact, experiments carried out at moderate heating rates $(10-20 \mathrm{~K} / \mathrm{min})$ or at isothermal conditions for a number of oxide precursors have always shown that their thin films do not experience combustion despite their powders do [18-20]. Furthermore, our theoretical analysis of the isoperibolic case delivered a lower bound for the critical thickness around $400 \mu \mathrm{m}$ [21]. To overcome this limitation, several authors $[14,16]$ have suggested that combustion may be easier to achieve when a substrate with its precursor film is put on a hot plate set at the processing temperature, i.e., the critical thickness is thought to be lower at very high heating rates. Now we are able to solve this question.

Consider that combustion of a precursor powder is observed by TG when it is heated at a moderate heating rate $b$ inside a crucible where it reaches height $H$. The onset of combustion will be close to the Kissinger temperature, $T_{K i s}$. The critical thickness of a thin film can be obtained from Eq. (13):

$$
H_{c r}=\sqrt{\frac{\delta_{c r}}{\theta_{T}} a t_{R}} .
$$

Substitution of $\theta_{T}$ and $t_{R}$ by their definitions, Eqs. (5) and (13), and application of Kissinger's equation, Eq. (6), leads to:

$$
H_{c r}=\frac{R_{G} T_{K i s}^{2}}{E_{A}} \sqrt{\delta_{c r} \frac{c a}{q} \frac{1}{b}} .
$$

Since $\sqrt{\delta_{c r}}$ will be the same within a factor of the order of 1 (see the evolution of $C_{C y l}^{(2 D)}$ in Fig. 5) when passing from a finite cylinder (powder inside a crucible) to a thin film, we can write the following relationship: 


$$
\frac{H}{H^{\prime}}=\sqrt{\frac{b^{\prime}}{b}}\left(\frac{T_{K i s}}{T_{K i s}{ }^{\prime}}\right)^{2} .
$$

Besides, $T_{\text {Kis }}$ scales as the logarithm of the heating rate $[55,56]$,

$$
\frac{T_{K i s}}{T_{K i s}{ }^{\prime}}=1+\frac{R_{G} T_{K i s}}{E_{A}} \ln \frac{b}{b^{\prime}} .
$$

Combining Eqs. (24) and (25) we obtain,

$$
\frac{H}{H^{\prime}}=\left(1+\frac{R_{G} T_{K i s}}{E_{A}} \ln \frac{b}{b^{\prime}}\right)^{2} \sqrt{\frac{b^{\prime}}{b}} .
$$

Suppose that a powder undergoes thermal explosion at, say, $b=10 \mathrm{~K} / \mathrm{min}$ and $H$ $=500 \mu \mathrm{m}$. For this heating rate, typical values of $E_{A} / R_{G} T_{K i s}$ are around 30 (this corresponds to $T_{K i \mathrm{~s}}=600 \mathrm{~K}$ for an activation energy of $150 \mathrm{~kJ} / \mathrm{mol}$ ). Thus, a thin film of $H^{\prime}=1 \mu \mathrm{m}$ will undergo a thermal runaway at a substrate temperature around $1222 \mathrm{~K}$ when heated at a rate as high as $410^{7} \mathrm{~K} / \mathrm{min}$. Therefore, low temperature synthesis of thin films is virtually impossible, even when films are heated at very high heating rates.

\section{Conclusions}

The partial differential equations (PDE) system describing heat transport during an exothermic reaction has been solved for 1D (infinite cylinder and thin film) and for 2D (finite cylinder) geometries when the vessel walls or substrate are heated at a constant rate.

It has been shown that, for large and small systems, ignition is produced close to its walls and at the center of the reactant volume, respectively, and that, when the reactant mass is small enough, no combustion occurs.

A systematic search of the critical condition of combustion has been undertaken for a very broad range of the three adimensional parameters (Ahrrenius, $\varepsilon$, Todes, $\theta_{\mathrm{T}}$, and Frank-Kamenetskii, $\delta$ ) that describe the system dynamics. The exact condition, $\delta_{c r}\left(\varepsilon, \theta_{T}\right)$, has been fitted to an analytical functional dependence that is valid for virtually all experimental situations. It has been shown that, in the limit of large activation energy $(\varepsilon \rightarrow 0)$ and high adiabatic temperature rise $\left(\theta_{T} \rightarrow \infty\right)$, the combustion critical condition is formally the same as that obtained for an isoperibolic system except for a factor 5 that indicates that, during constant heating conditions, a thermal runaway is more difficult to develop. 
This analytical condition has allowed us to derive a formula giving the critical mass for a cylindrical reactor of finite height. This formula agrees with the exact solution of the PDE system and with experimental results carried out with $\mathrm{Ba}(\mathrm{TFA})_{2}$.

In the case of thin films, it has been shown that extremely high heating rates are necessary to achieve combustion. Under this high heating rates, combustion occurs when the substrate reaches a high temperature, so low temperature thermal explosion is virtually impossible in thin films.

\section{Appendix A. Quasi-stationary approach.}

To derive the quasi-stationary and non-stationary models, we define a new variable that is the difference between the sample and the furnace temperatures,

$$
\vartheta(\tau, \xi) \equiv \theta(\tau, \xi)-\frac{\tau}{\theta_{T}}
$$

The evolution of $\vartheta$ is obtained by substituting Eq. (A.1) into Eqs. (10)-(12).

$$
\begin{aligned}
& \frac{\partial \vartheta}{\partial \tau}=\left(\frac{\partial^{2} \vartheta}{\partial \xi^{2}}+\frac{N}{\xi} \frac{\partial \vartheta}{\partial \xi}\right)+\theta_{T} \frac{\partial \alpha}{\partial \tau}-\frac{1}{\theta_{T}} . \\
& \frac{\partial \alpha}{\partial \tau}=\frac{1}{\theta_{T}} e^{\frac{\vartheta+\tau / \theta_{T}}{1+\varepsilon\left(\vartheta+\tau / \theta_{T}\right)}}(1-\alpha)
\end{aligned}
$$

with boundary conditions,

$$
\vartheta(\sqrt{\delta})=0 \text { and }\left.\frac{\partial \vartheta}{\partial \xi}\right|_{\xi=0}=0 .
$$

and initial conditions

$$
\vartheta_{i n}=0 \text { and } \tau_{i n}=-\frac{\theta_{T}}{\varepsilon},
$$

In the absence of a significant local overheating, the sample temperature will follow steadily the time dependence fixed by the furnace walls, i.e. $\partial T / \partial t=b$, and, consequently:

$$
\frac{\partial \theta}{\partial \tau} \approx \frac{1}{\theta_{T}} \Leftrightarrow \frac{\partial \vartheta}{\partial \tau} \approx 0 .
$$

Under this assumption and in the limit of $\varepsilon \rightarrow 0$ and $\theta_{T} \rightarrow \infty$ Eq. (A.2) becomes

$$
\left(\frac{\partial^{2} \vartheta}{\partial \xi^{2}}+\frac{N}{\xi} \frac{\partial \vartheta}{\partial \xi}\right)+(1-\alpha) e^{\vartheta} e^{\tau / \theta_{T}}=0 .
$$


To derive an analytical solution, we replace the actual value $\alpha$ for its average over the system volume, Eq. (14), i.e., we assume that $\alpha \approx \bar{\alpha}$. We define a new space coordinate as $\zeta=\sqrt{(1-\bar{\alpha}) e^{\tau / \theta_{T}}} \xi$. If we substitute $\xi$ by $\zeta$ in Eq. (A.6) we obtain

$$
\left(\frac{\partial^{2} \vartheta}{\partial \zeta^{2}}+\frac{N}{\zeta} \frac{\partial \vartheta}{\partial \zeta}\right)+e^{\vartheta}=0 .
$$

with boundary conditions

$$
\vartheta\left(\sqrt{\delta(1-\bar{\alpha}) e^{\tau / \theta_{T}}}\right)=0 \text { and }\left.\frac{\partial \vartheta}{\partial \xi}\right|_{\xi=0}=0 .
$$

Eqs. (A.7) and (A.8) and the stationary equation for the isoperibolic system $[21,30,52-$ 54] are nearly identical. The only difference lays in the first boundary condition where instead of Frank-Kamenetskii parameter, $\delta$, we have $\delta(1-\bar{\alpha}) e^{\tau / \theta_{T}}$.

Thus, in our case the temperature profiles coincide with those of the isoperibolic system. For instance, for $N=0$ (thin-film) the solution of Eqs. (A.7) and (A.8) is [21,53]

$$
\vartheta=2 \ln \left[k \operatorname{sech}\left(\frac{k}{\sqrt{2}} \zeta\right)\right] .
$$

where $k$ is an integration constant that is determined from the first boundary condition,

$$
\vartheta\left(\sqrt{\delta(1-\bar{\alpha}) e^{\tau / \theta_{T}}}\right)=0 \Leftrightarrow \delta(1-\bar{\alpha}) e^{\tau / \theta_{T}}=2\left(\frac{1}{k} \operatorname{arcosh}[k]\right)^{2} .
$$

For an infinite long cylinder $(N=1)$ the solution of Eqs. (A.7) and (A.8) is $[21,53]$

$$
\vartheta=2 \ln \left[\frac{4 k}{2 k^{2}+\zeta^{2}}\right] .
$$

To check the accuracy of the quasi-stationary solution, in Fig. A.1 we compare the temperature profiles calculated numerically with those delivered by the analytical solution, Eqs. (A.9) and (A.10), for a thin-film geometry. From Fig. A.1 it is clear that, at the first stages, the quasi-stationary solution provides an accurate description of the temperature profiles. However, near the onset of a thermal runaway ( $\bar{\alpha}=0.44)$, the deviations of the analytical solution are more apparent; when local overheating becomes significant, the assumption that $\alpha$ does not depend on position no longer holds.

Thermal explosion occurs when there is no solution for the quasi-stationary state, i.e., when Eq. (A.10) has no solution. Eq. (A.10) coincides with that of the static case but replacing $\delta$ by $\delta(1-\bar{\alpha}) e^{\tau / \theta_{T}}$ (Eq. (A.3) in [21]). Thus, in the limit $\varepsilon \rightarrow 0$ and 
$\theta_{T} \rightarrow \infty$, the critical condition under dynamic conditions is that of the static case [30], but replacing $\delta$ by $\delta(1-\bar{\alpha}) e^{\tau / \theta_{T}}$ :

$$
\left.\delta(1-\bar{\alpha}) e^{\tau / \theta_{T}}\right|_{c r}=C
$$

where subscript $\mathrm{cr}$ stands for the parameter value at the threshold of ignition, and $C=0.878$ and 2 , and $k_{c r}=1.81$ and 1 for a thin film and an infinite cylinder, respectively [21].

At the threshold, the thermal runaway will develop when the transformation rate at the center of the sample is at its maximum, i.e., when the center of the sample reaches the Kissinger temperature:

$$
\theta_{c r}(\zeta=0)=0 \Rightarrow \vartheta_{c r}(\zeta=0)=-\frac{\tau}{\theta_{T}}
$$

From the critical value of $k$ and from Eqs. (A.9), (A.11) and (A.13) we have determined the critical value of $\tau / \theta_{T}: \tau /\left.\theta_{T}\right|_{c r}=-1.18$ for a thin film and $\tau /\left.\theta_{T}\right|_{c r}=-1.38$ for an infinite cylinder. We have numerically calculated the values of $\bar{\alpha}_{c r}$ and $\tau /\left.\theta_{T}\right|_{c r}$ for the two extreme values of $\varepsilon(0$ and 0.1$)$; the results are plotted in Fig. A.2. From Fig. A.2 it is apparent that in the limit $\theta_{T} \rightarrow \infty, \tau /\left.\theta_{T}\right|_{c r} \rightarrow-1$. The same value is obtained irrespectively of the two geometries analyzed (thin film and infinite cylinder). The discrepancy between the analytical result and the numerical simulation is related to the fact that in the analytical analysis we have assumed $\alpha \approx \bar{\alpha}$ while $\alpha$ is not constant along the sample.

Once we know $\tau /\left.\theta_{T}\right|_{c r}$, we can determine the critical furnace temperature in the limit $\theta_{T} \rightarrow \infty$ :

$$
\theta_{f, c r}=-1 \Rightarrow T_{f, c r}=T_{K i s}(1-\varepsilon)
$$

Notice from Fig A.2 that, even for moderate values of $\theta_{T}, \tau /\left.\theta_{T}\right|_{c r} \approx-1$. Also, $\tau /\left.\theta_{T}\right|_{c r} \approx-1$ for $\varepsilon=0.1$. Indeed the numerical simulations show that for all the $\varepsilon$ values analyzed $\tau /\left.\theta_{T}\right|_{c r} \approx-1$. Thus Eq. (A.14) holds in a wide parameter range.

If we assume that $\tau /\left.\theta_{T}\right|_{c r} \approx-1$, from Eqs. (A.9) and (A.13) we obtain $k=\sqrt{e}$. If we substitute the value of $k$ in (A.9) we obtain the temperature distribution at the threshold, 


$$
\theta_{c r}=2 \ln \left[\sqrt{e} \operatorname{sech}\left(\sqrt{\frac{\mathrm{e}}{2} \zeta}\right)\right]-1, \quad 0 \leq \zeta \leq \sqrt{C} .
$$

From the definition of $\zeta$ and Eq. (A.12) we obtain that $\zeta=\sqrt{C}$ when $\xi=\sqrt{\delta}$, thus $\zeta$ ranges from 0 to $\sqrt{C}$.

From Eq. (10) and assuming Eq. (A.5) one can calculate $\alpha$ :

$$
\frac{\partial \alpha}{\partial \theta}=e^{\theta}(1-\alpha) \Rightarrow \alpha=1-e^{-e^{\theta}}
$$

Thus, once we know the temperature distribution we can determine the $\alpha$ distribution at the threshold:

$$
\alpha_{c r}=1-\exp \left[-\operatorname{sech}^{2}\left(\sqrt{\frac{\mathrm{e}}{2} \zeta}\right)\right], \quad 0 \leq \zeta \leq \sqrt{C} .
$$

Finally, to stablish the critical condition in the limit $\varepsilon \rightarrow 0$ and $\theta_{T} \rightarrow \infty$, Eq. (A.12), it is necessary to determine $\bar{\alpha}_{c r}$ :

$$
\bar{\alpha}_{c r}=\frac{1}{\sqrt{C}} \int_{0}^{\sqrt{C}} \alpha d \zeta=0.47 .
$$

This result agrees with the value that we have obtained from the integration of the PDE system, $\bar{\alpha}_{c r} \approx 0.44$. Finally, from the values of $\bar{\alpha}_{c r}$ and $\tau /\left.\theta_{T}\right|_{c r}$, we obtain $\left.(1-\bar{\alpha}) e^{\tau / \theta_{T}}\right|_{c r} \approx 1 / 5$, and the critical condition under constant heating in the limit $\varepsilon \rightarrow 0$ and $\theta_{T} \rightarrow \infty$ reduces to:

$$
\delta_{c r}=5 C \text {. }
$$

\section{Appendix B. Non-stationary approach}

In Appendix $\mathrm{A}$ we have stated that the temperature profiles under isoperibolic conditions and under constant heating conditions are similar. Therefore, like in the isoperibolic case, we can approximate the Laplacian term to $-B \vartheta / \delta$ :

$$
\frac{d \vartheta}{d \tau}=-\frac{B}{\delta} \vartheta+\theta_{T} \frac{d \alpha}{d \tau}+\frac{1}{\theta_{T}} .
$$

where $B$ is a geometrical factor [21],

$$
B=e \cdot C .
$$

Finally, if we substitute $\vartheta$ by $\theta$ we obtain the non-stationary ODE system, 


$$
\begin{aligned}
& \frac{d \theta}{d \tau}=-\frac{e C}{\delta}\left(\theta-\frac{\tau}{\theta_{T}}\right)+\theta_{T} \frac{d \alpha}{d \tau} \\
& \frac{d \alpha}{d \tau}=\frac{1}{\theta_{T}} e^{\frac{\theta}{1+\varepsilon \theta}}(1-\alpha)
\end{aligned} .
$$

With initial conditions,

$$
\theta_{i n}=-\frac{1}{\varepsilon} \text { and } \tau_{i n}=-\frac{\theta_{T}}{\varepsilon}
$$

\section{Acknowledgements}

This work was partially funded by the Spanish Programa Nacional de Materiales through project MAT2014-51778-C2-2-R, by the Generalitat de Catalunya contract No. 2014SGR-00948 and by the Universitat de Girona contract No. MPCUdG2016/059. 


\section{References}

[1] J. Adler, J.W. Enig, The critical conditions in thermal explosion theory with reactant consumption, Combust. Flame. 8 (1964) 97-103.

[2] J.M. Zaldívar, J. Cano, M.A. Alós, J. Sempere, R. Nomen, D. Lister, G. Maschio, T. Obertopp, E.D. Gilles, J. Bosch, F. Strozzi, A general criterion to define runaway limits in chemical reactors, J. Loss Prev. Process Ind. 16 (2003) 187200 .

[3] B. Roduit, L. Xia, P. Folly, B. Berger, J. Mathieu, A. Sarbach, H. Andres, M. Ramin, B. Vogelsanger, D. Spitzer, H. Moulard, D. Dilhan, The simulation of the thermal behavior of energetic materials based on DSC and HFC signals, J. Therm. Anal. Calorim. 93 (2008) 143-152.

[4] G. Krause, Volume-Dependent Self-Ignition Temperatures for Explosive Materials, Propellants, Explos. Pyrotech. 37 (2012) 107-115.

[5] B. Roduit, M. Hartmann, P. Folly, A. Sarbach, P. Brodard, R. Baltensperger, Determination of thermal hazard from DSC measurements. Investigation of selfaccelerating decomposition temperature (SADT) of AIBN, J. Therm. Anal. Calorim. 117 (2014) 1017-1026.

[6] M. Dellavedova, C. Pasturenzi, L. Gigante, A. Lunghi, Kinetic Evaluations for the Transportation of Dangerous Chemical Compounds, Chem. Eng. Trans. 26 (2012) 585-590.

[7] T. Boddington, A. Cottrell, P.G. Laye, A numerical model of combustion in gasless pyrotechnic systems, Combust. Flame. 76 (1989) 63-69.

[8] J. Zinn, C.L. Mader, Thermal Initiation of Explosives, J. Appl. Phys. 31 (1960) 323.

[9] R.N. Rogers, Thermochemistry of explosives, Thermochim. Acta. 11 (1975) $131-139$.

[10] A.C. Victor, Simple Calculation Methods for Munitions Cookoff times and temperatures, Propellants, Explos. Pyrotech. 20 (1995) 252-259.

[11] T. Kotoyori, Critical Temperatures for the Thermal Explosion of Chemicals, 1st ed., Elsevier, Amsterdam, 2005.

[12] J.F. Griffiths, J.A. Barnard, Flame and Combustion, 3rd ed., CRC Press, London, 1995.

[13] K. Morsi, The diversity of combustion synthesis processing: a review, J. Mater. 
Sci. 47 (2011) 68-92.

[14] A. Varma, A.S. Mukasyan, A.S. Rogachev, K. V. Manukyan, Solution Combustion Synthesis of Nanoscale Materials, Chem. Rev. 116 (2016) 1449314586.

[15] M.-G. Kim, M.G. Kanatzidis, A. Facchetti, T.J. Marks, Low-temperature fabrication of high-performance metal oxide thin-film electronics via combustion processing., Nat. Mater. 10 (2011) 382-8.

[16] W. Marchal, C. De Dobbelaere, J. Kesters, G. Bonneux, J. Vandenbergh, H. Damm, T. Junkers, W. Maes, J. D’Haen, M.K. Van Bael, A. Hardy, Combustion deposition of MoO 3 films: from fundamentals to OPV applications, RSC Adv. 5 (2015) 91349-91362.

[17] D. Sanchez-Rodriguez, J. Farjas, P. Roura, S. Ricart, N. Mestres, X. Obradors, T. Puig, Thermal Analysis for Low Temperature Synthesis of Oxide Thin Films from Chemical Solutions, J. Phys. Chem. C. 117 (2013) 20133-20138.

[18] P. Roura, J. Farjas, H. Eloussifi, L. Carreras, S. Ricart, T. Puig, X. Obradors, Thermal analysis of metal organic precursors for functional oxide preparation: Thin films versus powders, Thermochim. Acta. 601 (2015) 1-8.

[19] H. Eloussifi, J. Farjas, P. Roura, S. Ricart, T. Puig, X. Obradors, M. Dammak, Thermal decomposition of barium trifluoroacetate thin films, Thermochim. Acta. 556 (2013) 58-62.

[20] H. Eloussifi, J. Farjas, P. Roura, S. Ricart, T. Puig, X. Obradors, M. Dammak, Thermoanalytical study of the decomposition of yttrium trifluoroacetate thin films, Thin Solid Films. 545 (2013) 200-204.

[21] D. Sánchez-Rodriguez, J. Farjas, P. Roura, The critical condition for thermal explosion in an isoperibolic system, AIChE J. 63 (2017) 3979-3993.

[22] J. Farjas, D. Sánchez-Rodriguez, H. Eloussifi, P. Roura, Thermal Gradients in Thermal Analysis Experiments, in: J. Šesták, P. Hubík, J.J. Mareš (Eds.), Therm. Phys. Therm. Anal. From Macro to Micro, Highlighting Thermodyn. Kinet. Nanomater., Budapest, 2017: pp. 345-362.

[23] A.G. Merzhanov, A.G. Strunina, Laws of thermal explosion with constant heating rate, Combust. Explos. Shock Waves. 1 (1965) 43-52.

[24] V. V. Barzykin, Thermal explosion under linear heating, Combust. Explos. Shock Waves. 9 (1973) 29-42.

[25] A.G. Merzhanov, V.V. Barzykin, A.S. Shteinberg, V.T. Gontkovskaya, 
Methodological Principles in studying chemical reaction kinetics under conditions of programmed heating, Thermochim. Acta. 21 (1977) 301-332.

[26] I.S. Lyubchenko, V.N. Kosolapov, Approximate methods of determining the characteristics in the problem of a thermal explosion under linear heating conditions, Combust. Explos. Shock Waves. 14 (1978) 202-210.

[27] A.G. Merzhanov, V.V. Barzykin, V.G. Abramov, The theory of thermal explosion: From N.N.Semenov to present day, Chem. Phys. Reports. 15 (1996).

[28] A.G. Strunina, V.T. Gontkovskaya, A.G. Merzhanov, Laws of thermal explosion, Combust. Explos. Shock Waves. 1 (1965) 20-22.

[29] T. Boddington, F. Hongtu, P.G. Laye, M. Nawaz, D.C. Nelson, Thermal runaway by thermal analysis, Thermochim. Acta. 170 (1990) 81-87.

[30] D.A. Frank-Kamenetskii, Diffusion and Heat Exchange in Chemical Kinetics, 2nd ed., Princeton University Press, New Jersey, 1955.

[31] A.G. Merzhanov, A.E.E. Averson, The present state of the thermal ignition theory: An invited review, Combust. Flame. 16 (1971) 89-124.

[32] A.G. Merzhanov, B.I. Khaikin, Theory of combustion waves in homogeneous media, Prog. Energy Combust. Sci. 14 (1988) 1-98.

[33] S. Hwang, A.S. Mukasyan, A. Varma, Mechanisms of combustion wave propagation in heterogeneous reaction systems, Combust. Flame. 115 (1998) $354-363$.

[34] T. Fiedler, I. V Belova, S. Broxtermann, G.E. Murch, A thermal analysis on selfpropagating high temperature synthesis in joining technology, Comput. Mater. Sci. 53 (2012) 251-257.

[35] W. Squire, A mathematical analysis of self-ignition, Combust. Flame. 7 (1963) $1-8$.

[36] A.S. Mukasyan, A.S. Rogachev, Discrete reaction waves: Gasless combustion of solid powder mixtures, Prog. Energy Combust. Sci. 34 (2008) 377-416.

[37] E. García, D. Sánchez-Rodríguez, J.P. López-Olmedo, J. Farjas, P. Roura, The effect of volatiles on the measurement of the reaction heat by differential scanning calorimetry, J. Therm. Anal. Calorim. (2015) 187-194.

[38] J. Van’t Hoff, Études de dynamique chimique, Frederik Müller et Co, Amsterdam, 1884.

[39] S. Arrhenius, Über die Reaktionsgeschwindigkeit bei der Inversion von Rohrzucker durch Säuren, Zeitschrift Für Phys. Chemie. 4 (1889) 226-48. 
[40] J. Farjas, P. Roura, Simple approximate analytical solution for nonisothermal single-step transformations: Kinetic analysis, AIChE J. 54 (2008) 2145-2154.

[41] J. Farjas, N. Butchosa, P. Roura, A simple kinetic method for the determination of the reaction model from non-isothermal experiments, J. Therm. Anal. Calorim. 102 (2010) 615-625.

[42] H.E. Kissinger, Reaction Kinetics in Differential Thermal Analysis, Anal. Chem. 29 (1957) 1702-1706.

[43] P. Budrugeac, E. Segal, Applicability of the kissinger equation in thermal analysis revisited, J. Therm. Anal. Calorimetry; 9th Eur. Symp. Therm. Anal. Calorim. 88 (2007) 703-707.

[44] O.M. Todes, P. V Melentjew, The theory of heat explosion II Heat explosion for mono-molecular reactions, Acta Physicochim. URSS. 11 (1939) 153-180.

[45] J. Farjas, J. Camps, P. Roura, S. Ricart, T. Puig, X. Obradors, The thermal decomposition of barium trifluoroacetate, Thermochim. Acta. 544 (2012) 77-83.

[46] A. Khawam, D.R. Flanagan, Basics and applications of solid-state kinetics: a pharmaceutical perspective., J. Pharm. Sci. 95 (2006) 472-98.

[47] D. Sánchez-Rodríguez, H. Eloussifi, J. Farjas, P. Roura, M. Dammak, Thermal gradients in thermal analysis experiments: Criterions to prevent inaccuracies when determining sample temperature and kinetic parameters, Thermochim. Acta. 589 (2014) 37-46.

[48] D. Sánchez-Rodríguez, H. Wada, S. Yamaguchi, J. Farjas, H. Yahiro, Selfpropagating high-temperature synthesis of LaMO3 perovskite-type oxide using heteronuclearcyano metal complex precursors, J. Alloys Compd. 649 (2015) 1291-1299.

[49] J. Zinn, R.N. Rogers, Thermal initiation of explosives 1, J. Phys. Chem. 66 (1962) 2646-2653.

[50] H. Eloussifi, J. Farjas, P. Roura, J. Camps, M. Dammak, S. Ricart, T. Puig, X. Obradors, Evolution of yttrium trifluoroacetate during thermal decomposition, $\mathrm{J}$. Therm. Anal. Calorim. 108 (2012) 589-596.

[51] T. Striker, J.A. Ruud, Effect of Fuel Choice on the Aqueous Combustion Synthesis of Lanthanum Ferrite and Lanthanum Manganite, J. Am. Ceram. Soc. 93 (2010) 2622-2629.

[52] P.L. Chambré, On the Solution of the Poisson-Boltzmann Equation with Application to the Theory of Thermal Explosions, J. Chem. Phys. 20 (1952) 
1795.

[53] W. Gill, A.B. Donaldson, A.R. Shouman, The Frank-Kamenetskii problem revisited. Part I. Boundary conditions of first kind, Combust. Flame. 36 (1979) $217-232$.

[54] C. Harley, E. Momoniat, Alternate Derivation of the Critical Value of the FrankKamenetskii Parameter in Cylindrical Geometry, J. Nonlinear Math. Phys. 15 (2008) 69-76.

[55] P. Roura, J. Farjas, Analytical solution for the Kissinger equation, J. Mater. Res. 24 (2009) 3095-3098.

[56] J. Farjas, P. Roura, Exact analytical solution for the Kissinger equation: Determination of the peak temperature and general properties of thermally activated transformations, Thermochim. Acta. 598 (2014) 51-58. 
Table 1. Physical parameters of the metal organic powder analyzed.

\begin{tabular}{lr}
\hline & $\mathrm{Ba}(\mathrm{TFA})_{2}$ \\
\hline Thermal conductivity, $\lambda, \mathrm{W} /(\mathrm{m} \cdot \mathrm{K})$ & 0.08 \\
Specific heat capacity, $c, \mathrm{~J} /(\mathrm{kg} \cdot \mathrm{K})$ & 2230 \\
Density, $\rho, \mathrm{kg} / \mathrm{m}^{3}$ & 1463 \\
Specific heat of reaction, $q, \mathrm{~J} / \mathrm{kg}$ & $1.0 \times 10^{6}$ \\
Activation energy, $E_{A}, \mathrm{~J} / \mathrm{mol}$ & $1.75 \times 10^{5}$ \\
Pre-exponential constant, $A, \mathrm{~s}^{-1}$ & $4.5 \times 10^{13}$ \\
\hline
\end{tabular}

Table 2. Physical parameters of the alumina crucibles.

\begin{tabular}{lr}
\hline & $\mathrm{Al}_{2} \mathrm{O}_{3}$ \\
\hline Thermal conductivity, $\lambda, \mathrm{W} /(\mathrm{m} \cdot \mathrm{K})$ & 39 \\
Specific heat capacity, $c, \mathrm{~J} /(\mathrm{kg} \cdot \mathrm{K})$ & 775 \\
Density, $\rho, \mathrm{kg} / \mathrm{m}^{3}$ & 3980 \\
Thermal diffusivity, $\mathrm{m}^{2} / \mathrm{s}$ & $1.3 \times 10^{-5}$ \\
\hline
\end{tabular}




\section{Figure captions}

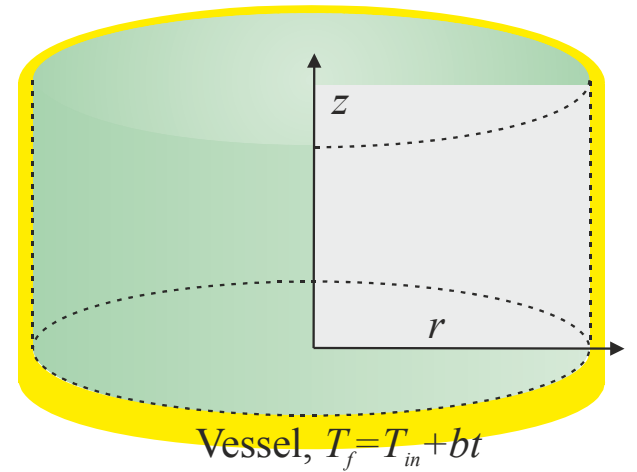

Figure 1. Geometry analyzed. The sample is placed inside a cylindrical vessel without a cover.

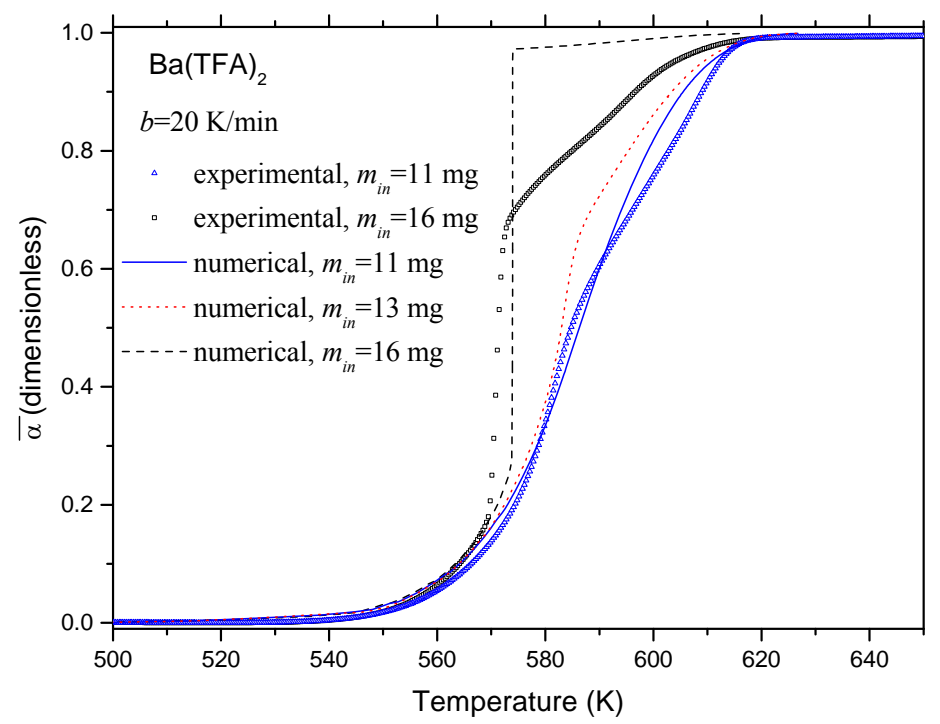

Figure 2. Experimental (symbols) and numerical evolution of the thermal evolution of $\mathrm{Ba}(\mathrm{TFA})_{2}$ powder inside a cylindrical alumina crucible (internal and external radii of 2.4 and $3 \mathrm{~mm}$, respectively). Experimental data corresponds to TG measurements. The furnace temperature is raised from 293 at a rate of $20 \mathrm{~K} / \mathrm{min}$ in wet synthetic air. 


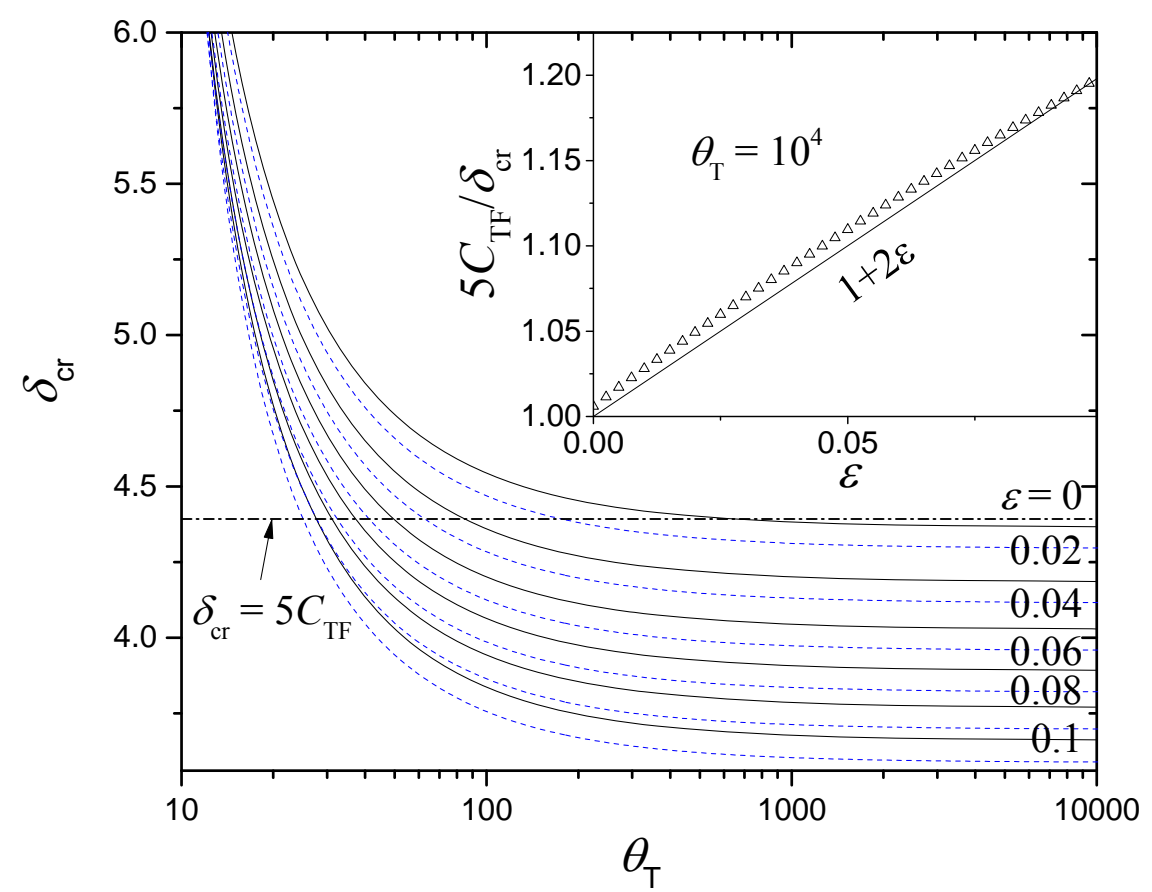

Figure 3 Values of $\delta_{c r}$ determined numerically assuming thin film geometry for different values of the dimensionless parameters $\theta_{T}$ and $\varepsilon$. Solid line: obtained from the exact model, Eqs. (10)-(12). Dashed line: obtained from the ODE system, Eq. (15). The horizontal line corresponds to the limit value for $\varepsilon=0$ and $\theta_{T} \rightarrow \infty$ predicted by the quasi-stationary approach, Eq. (16). Inset: fitted dependence on $\varepsilon$ for $\theta_{T} \rightarrow \infty$. 


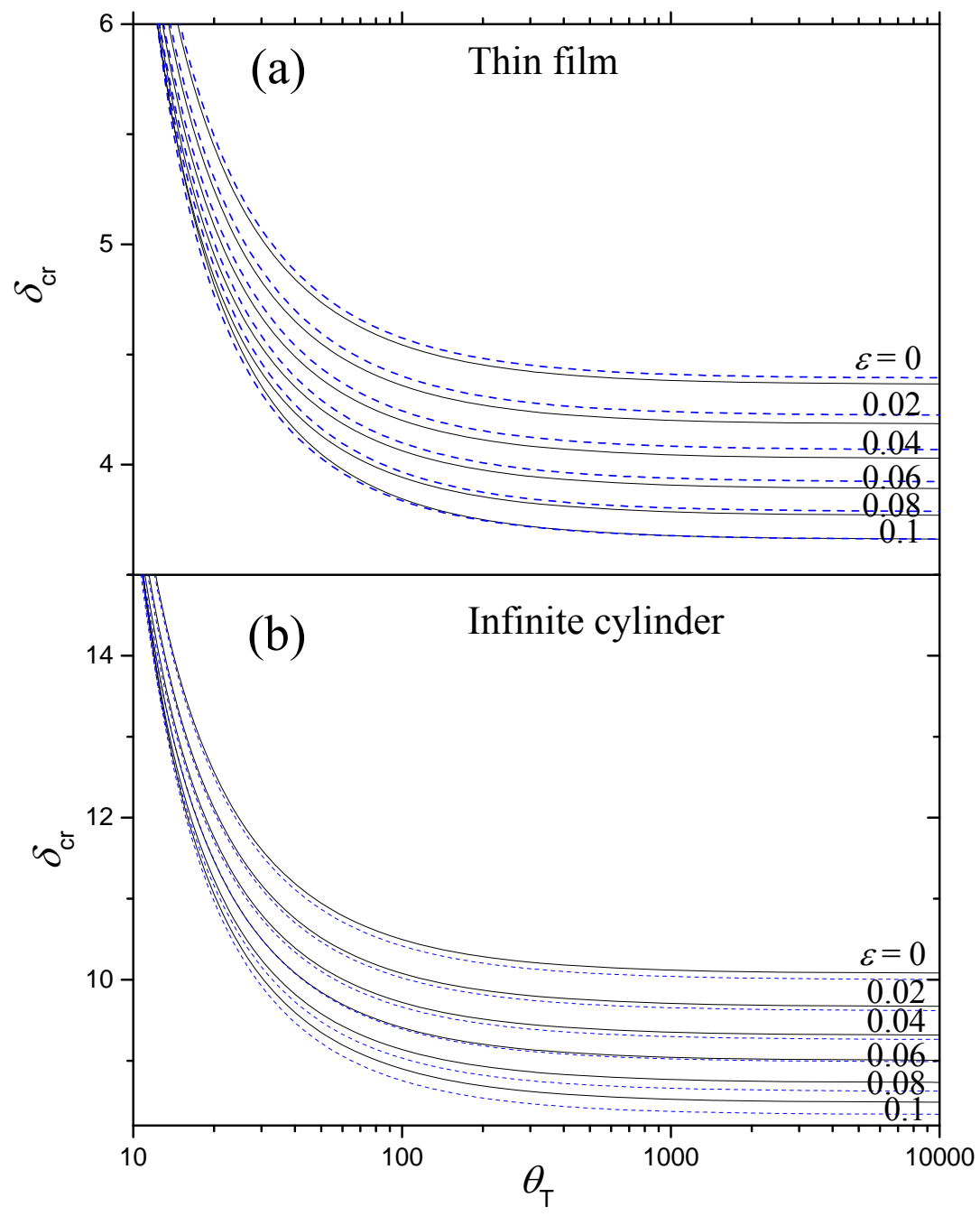

Figure 4. Values of $\delta_{c r}$ determined numerically assuming thin film (a) and infinite cylinder (b) geometries. Solid line: integration of the exact model, Eqs. (10)-(12). Dashed line: prediction of the critical condition, Eq. (18). 


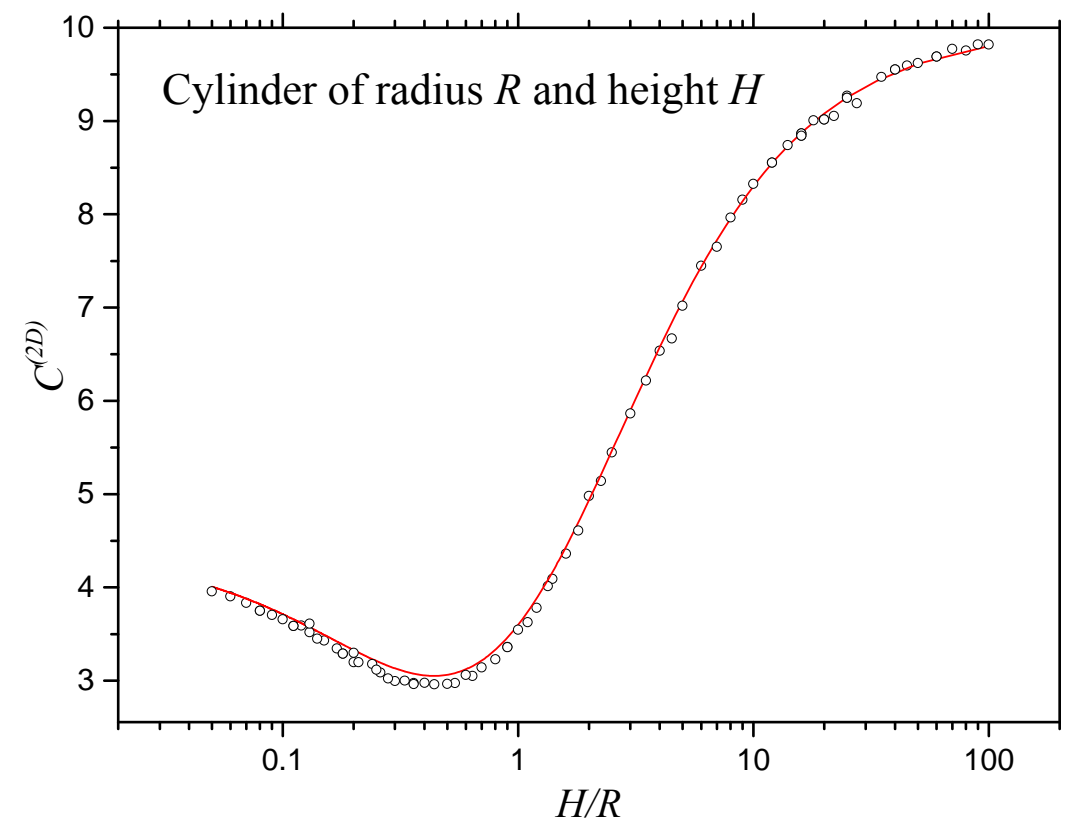

Figure 5. Geometrical factor, $C_{C y l}^{(2 D)}$, for a cylinder of radius $R$ and height $H$, determined from an isoperibolic system, Eq. (19), (solid line) and determined from Eq. (18) (symbols) for given values of $\delta, \varepsilon$, and $\theta_{\mathrm{T}}$ at the threshold of thermal runaway. The values of $\delta, \varepsilon$, and $\theta_{\mathrm{T}}$ at the threshold of thermal runaway have been determined from the numerical integration of the exact 2D model. 


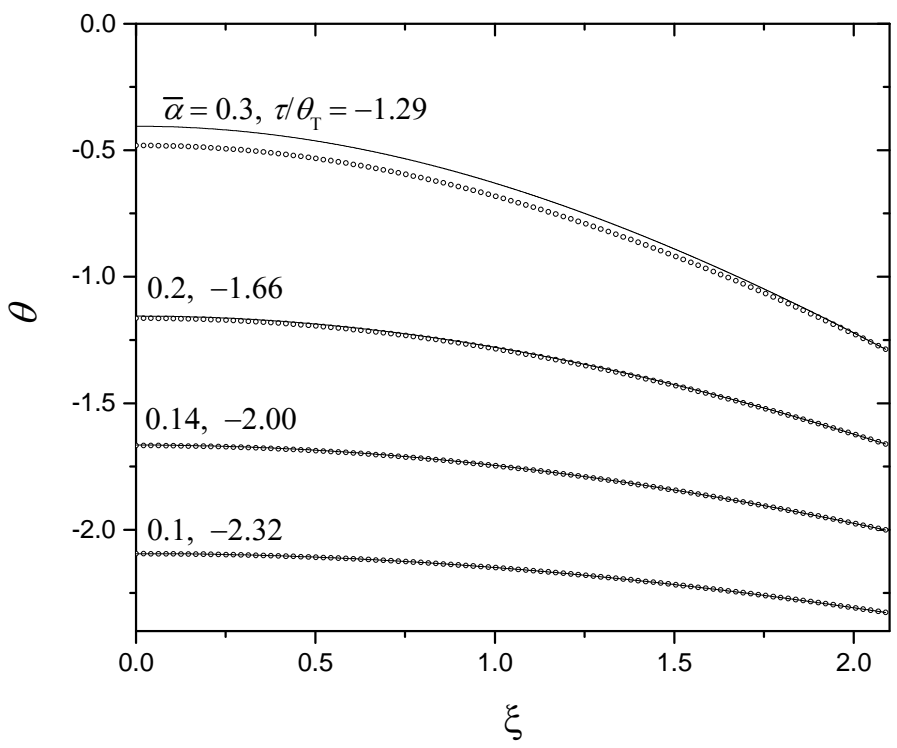

Figure A1. Evolution of the temperature profiles near the threshold of thermal explosion $(\bar{\alpha}=0.44)$ for a film. Symbols: numerical integration of Eqs. (10)-(12) for $\delta=4.365, \theta_{\mathrm{T}}=8 \cdot 10^{5}$ and $\varepsilon=0$. Solid line: analytical solution, Eqs (A.9) and (A.11). 


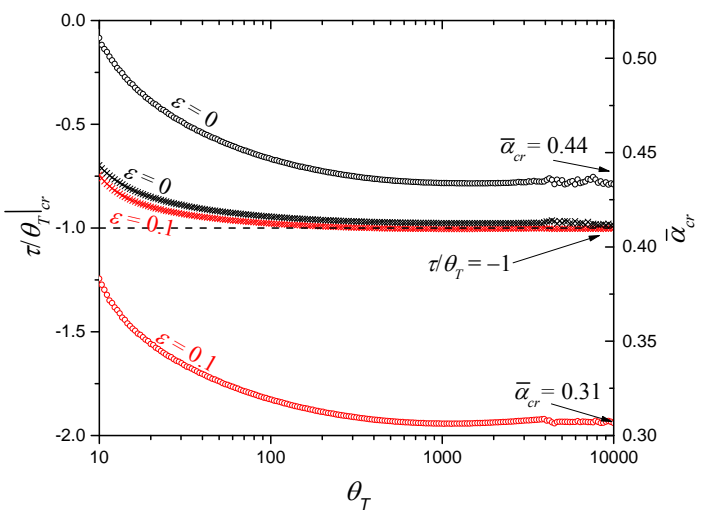

Figure A2. . Values of $\bar{\alpha}_{c r}$ (circles) and $\tau /\left.\theta_{T}\right|_{c r}$ (crosses) determined numerically from the integration of the exact model for a film, Eqs. (10)-(12) for $\varepsilon=0$ (black) and $\varepsilon=0.1$ (red). 


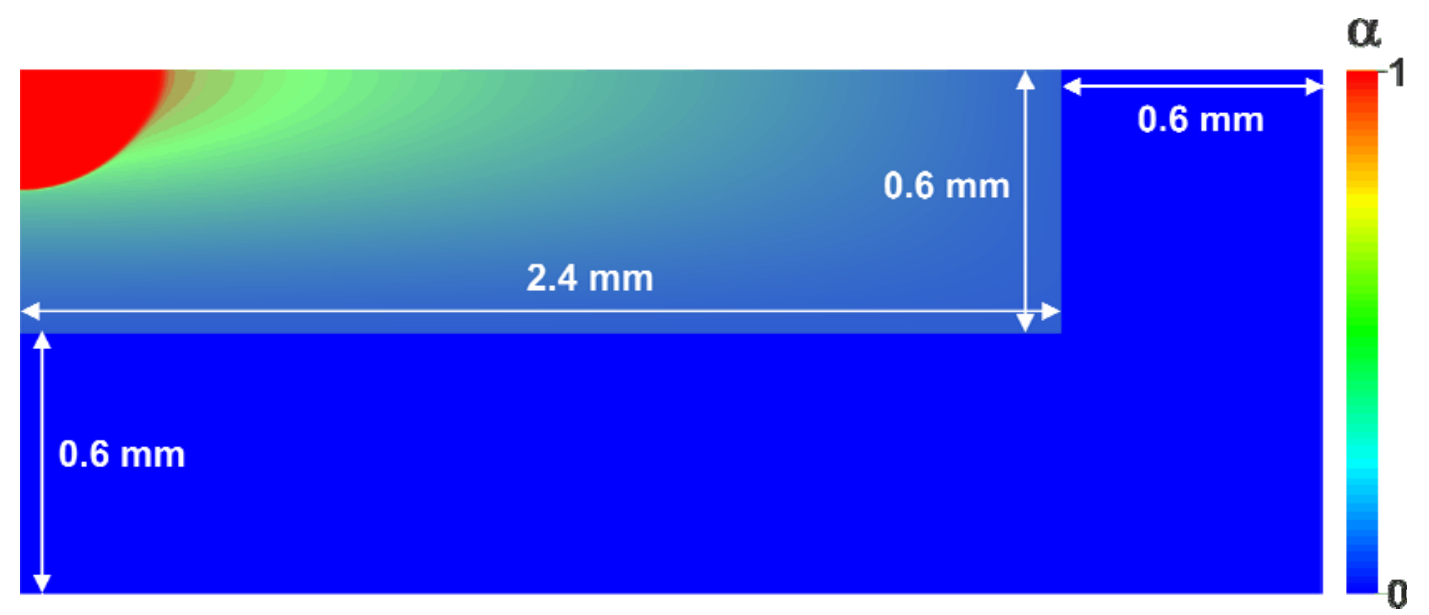

Movie 1. Evolution of the reaction for $\mathrm{Ba}(\mathrm{TFA})_{2}$ powders inside an alumina crucible. The physical parameters are those of Fig. 2 (internal and external radii of 2.4 and $3 \mathrm{~mm}$, respectively, $m_{\text {in }}=16 \mathrm{mg}$ ).

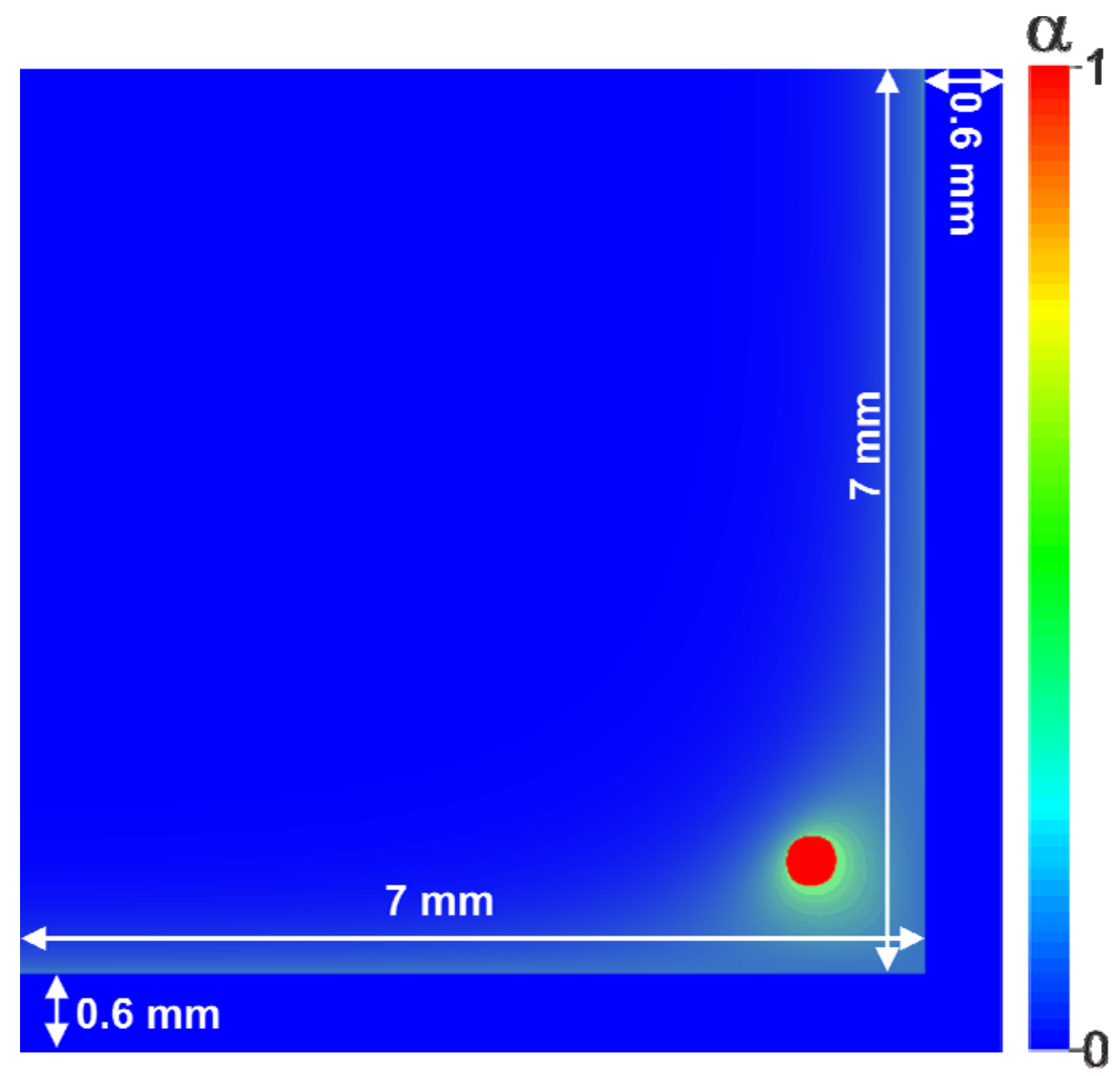

Movie 2. Evolution of the reaction for $\mathrm{Ba}(\mathrm{TFA})_{2}$ powders inside an alumina crucible. Simulation parameters coincide with those of Figure 2 except for the dimensions of the system: $H=R=7 \mathrm{~mm}$ and the vessel wall thickness is $0.6 \mathrm{~mm}$. 


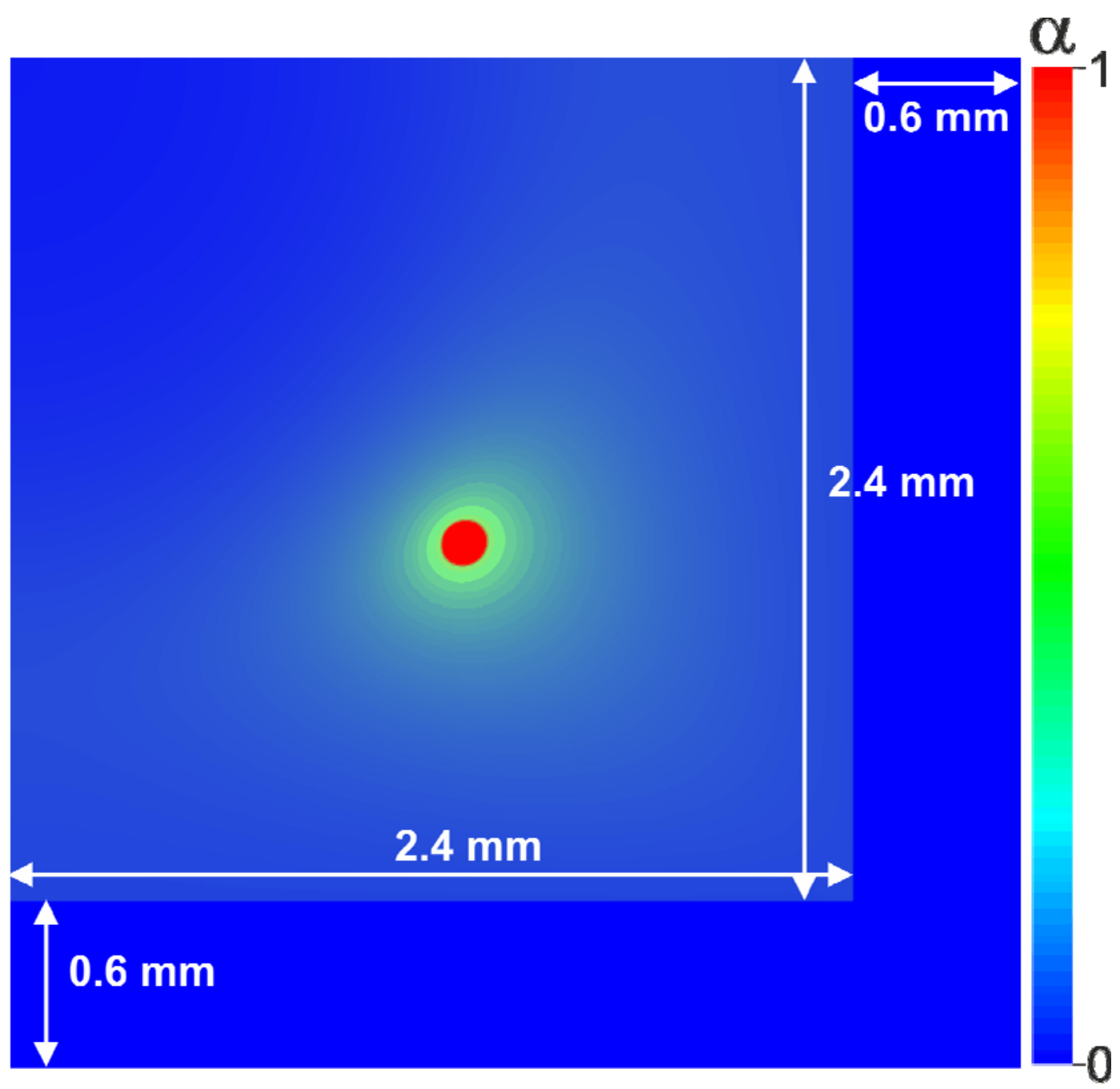

Movie 3. Evolution of the reaction for $\mathrm{Ba}(\mathrm{TFA})_{2}$ powders inside an alumina crucible. Simulation parameters coincide with those of Figure 2 except for the dimensions of the system: $H=R=2.4 \mathrm{~mm}$ and the vessel wall thickness is $0.6 \mathrm{~mm}$. 